\title{
Spinning up asymptotically flat spacetimes
}

\author{
E N Glass and J P Krisch \\ Department of Physics, University of Michigan, Ann Arbor, MI 48109, USA
}

Received 26 January 2004, in final form 23 September 2004

Published 16 November 2004

Online at stacks.iop.org/CQG/21/5543

doi:10.1088/0264-9381/21/23/015

\begin{abstract}
We present a method for constructing stationary, asymptotically flat, rotating solutions of Einstein's field equations. One of the spun-up solutions has quasilocal mass but no global mass. It has an ergosphere but no event horizon. The angular momentum is constant everywhere beyond the ergosphere. The energy-momentum content of this solution can be interpreted as a rotating string fluid.
\end{abstract}

PACS numbers: $04.20 . J b, 04.40 . \mathrm{Dg}$

\section{Introduction}

A metric description of the spacetime around an object encodes information about its mass and angular momentum. While many of the objects of interest to astrophysicists and cosmologists are rotating, there is not a large number of metrics that can be used to describe isolated rotating objects. Most models of the early universe encode no angular momentum into the metric. Developing methods to generate rotating solutions is clearly of value. There are two well-known solution generating algorithms which start with a static metric and transform it by adding rotation to some aspect of the spacetime structure. The complex transformation discovered by Newman (N-J) and others [1-3], for example, takes the Schwarzschild metric to the Kerr metric, and the Reissner-Nordström metric to the Kerr-Newman solution. The $\mathrm{N}-\mathrm{J}$ transform adds angular momentum to a seed spacetime initially containing only global mass (and possibly charge). The $\mathrm{N}-\mathrm{J}$ method has also been used [4] to spin up spherical metrics to obtain Kerr interiors. There is a method begun by Ehlers [5] and fully developed by Geroch [6, 7] which adds twist to the timelike Killing vector of the original (seed) metric and also changes its norm. If the method is applied to the static Schwarzschild spacetime the Taub-Nut spacetime results.

In this paper, we suggest a new method for adding rotation to a known static spacetime. The method starts with an asymptotically flat static metric written in null Bondi-Sachs form. A transform of the type $\mathrm{d} \tilde{\varphi}=\mathrm{d} \varphi-\Omega \mathrm{d} u$ is applied. $\Omega$ has coordinate dependence thus adding angular momentum to the spacetime. The Komar integral is used to calculate the angular momentum. Asymptotic flatness is enforced by using the Bondi-Sachs metric as a 
prototype for adding angular momentum, with the Bondi-Sachs metric conditions ensuring that asymptotic flatness is preserved in the transformation.

In the next section, we describe the method in detail. In section 3 of the paper we apply the method to the Schwarzschild spacetime, adding a rotation term, maintaining the Bondi-Sachs metric form and using the rotation term from the linearized Kerr metric in the Bondi-Sachs frame as a guide. We also apply the method to Minkowski space, obtainable as a limiting case of the spinning Schwarzschild example. This spacetime contains some unusual features. Unusual because there is quasilocal mass but no global mass, and there is an ergosphere but no event horizon. The solution is stationary, axially symmetric and asymptotically flat. The energy-momentum content can be identified as a rotating string fluid. Values from the Komar superpotential provide angular momentum which does not fall off asymptotically and quasilocal mass which does. The quasilocal mass arises from the kinetic energy of the rotating string fluid.

\section{Conventions}

In this work Greek indices range over $(0,1,2,3)=(u, r, \vartheta, \varphi)$. Sign conventions are $2 A_{v ;[\alpha \beta]}=A_{\mu} R^{\mu}{ }_{\nu \alpha \beta}$ and $R_{\alpha \beta}=R_{\alpha \beta \nu}^{v}$. The metric signature is $(+,-,-,-)$ and the field equations are $G_{\mu \nu}=-8 \pi T_{\mu \nu}$.

\section{The method}

The Bondi-Sachs metric has been used since 1960s. It is based on a foliation of outgoing null hypersurfaces $(\mathcal{N})$ and a conformal boundary $(\mathcal{I})$, with sufficient generality to describe bounded radiating astrophysical systems (details are given in appendix D). Asymptotically flat electrovac spacetimes in Bondi-Sachs form are summarized by Bicak and Pravdova [8].

To construct our solution, we begin with the Schwarzschild vacuum metric. Angular momentum is added using the $\partial_{\varphi}$ Killing vector as a generator of rotations. This is done in the context of the Bondi-Sachs metric in order to identify the term to add, and to maintain asymptotic flatness. A new term is added containing angular velocity with form $\mathrm{d} \tilde{\varphi}=\mathrm{d} \varphi-\Omega \mathrm{d} u$. For $\Omega$ a function rather than a constant, this is not an integrable coordinate transformation. The Komar superpotential integral for $\partial_{\varphi}$ insures that the desired amount of angular momentum is added. The particular choice of $\Omega$ is guided by the linearized Kerr metric written in Bondi-Sachs form. We then have an analytic asymptotically flat spinning metric.

\subsection{Kerr vacuum solution}

The Kerr solution in Boyer-Lindquist coordinates is given by

$$
\begin{aligned}
g_{\alpha \beta}^{\text {kerr }} \mathrm{d} x^{\alpha} \mathrm{d} x^{\beta}= & \psi \mathrm{d} t^{2}-(\Sigma / \Delta) \mathrm{d} r^{2}+(1-\psi) 2 a \sin ^{2} \vartheta \mathrm{d} t \mathrm{~d} \varphi \\
& -\Sigma \mathrm{d} \vartheta^{2}-\sin ^{2} \vartheta\left[\Sigma+(2-\psi) a^{2} \sin ^{2} \vartheta\right] \mathrm{d} \varphi^{2}
\end{aligned}
$$

Here, $\psi=1-2 m_{0} r / \Sigma, \Sigma=r^{2}+a^{2} \cos ^{2} \vartheta, \Delta=r^{2}+a^{2}-2 m_{0} r$. The linearized Kerr metric has terms linear in ' $a$ '.

$$
\begin{aligned}
g_{\alpha \beta}^{\text {lin-Kerr }} \mathrm{d} x^{\alpha} \mathrm{d} x^{\beta} & =\left(1-2 m_{0} / r\right) \mathrm{d} t^{2}-\left(1-2 m_{0} / r\right)^{-1} \mathrm{~d} r^{2} \\
+ & \left(2 m_{0} a / r\right) \sin ^{2} \vartheta 2 \mathrm{~d} t \mathrm{~d} \varphi-r^{2}\left(\mathrm{~d} \vartheta^{2}+\sin ^{2} \vartheta\right) \mathrm{d} \varphi^{2} .
\end{aligned}
$$


To reach Bondi-Sachs form transform from Boyer-Lindquist ' $t$ ' to outgoing $u=t-r-$ $2 m_{0} \ln \left(r-2 m_{0}\right)$.

$$
\begin{aligned}
& g_{\alpha \beta}^{\text {lin-Kerr-BS }} \mathrm{d} x^{\alpha} \mathrm{d} x^{\beta}=\left(1-2 m_{0} / r\right) \mathrm{d} u^{2}+2 \mathrm{~d} u \mathrm{~d} r+\left(2 m_{0} a / r\right) \sin ^{2} \vartheta 2 \mathrm{~d} u \mathrm{~d} \varphi \\
&-\left(1-2 m_{0} / r\right)^{-1}\left(2 m_{0} a / r\right) \sin ^{2} \vartheta 2 \mathrm{~d} r \mathrm{~d} \varphi-r^{2}\left(\mathrm{~d} \vartheta^{2}+\sin ^{2} \vartheta\right) \mathrm{d} \varphi^{2} .
\end{aligned}
$$

Note that

$$
(\mathrm{d} u)_{\alpha}(\mathrm{d} u)_{\beta} g_{\text {Kerr }}^{\alpha \beta}=0 \text { to order ' } a \text { '. }
$$

\section{Spinning Schwarzschild}

The Schwarzschild vacuum is spun up by choosing a term with angular velocity $\Omega(r)$. The spinning metric is written in Bondi-Sachs form as

$g_{\alpha \beta}^{\mathrm{BS}} \mathrm{d} x^{\alpha} \mathrm{d} x^{\beta}=\left(1-2 m_{0} / r\right) \mathrm{d} u^{2}+2 \mathrm{~d} u \mathrm{~d} r-r^{2} \mathrm{~d} \vartheta^{2}-r^{2} \sin ^{2} \vartheta(\mathrm{d} \varphi-\Omega \mathrm{d} u)^{2}$.

The term $\left(2 m_{0} a / r\right) \sin ^{2} \vartheta 2 \mathrm{~d} u \mathrm{~d} \varphi$ in the linearized Kerr metric equation (2) leads to the function $U^{\varphi}=\Omega(r)$ in equation (D.1), multiplied by $r^{2} H_{\varphi \varphi}=r^{2} \sin ^{2} \vartheta$. This directs the choice $\Omega(r)=r_{0}^{2} / r^{3}$.

The Bondi-Sachs form can be rewritten as the spinning Schwarzschild (sSCH) metric

$g_{\alpha \beta}^{\mathrm{sSCH}} \mathrm{d} x^{\alpha} \mathrm{d} x^{\beta}=Z \mathrm{~d} u^{2}+2 \mathrm{~d} u \mathrm{~d} r+2 \Omega r^{2} \sin ^{2} \vartheta \mathrm{d} u \mathrm{~d} \varphi-r^{2}\left(\mathrm{~d} \vartheta^{2}+\sin ^{2} \vartheta \mathrm{d} \varphi^{2}\right)$

with $Z:=1-2 m_{0} / r-(\Omega r \sin \vartheta)^{2}$.

To understand the physical content of the $\mathrm{SSCH}$ solution we write the metric in a basis which is locally non-rotating $\left(\hat{v}^{\alpha}\right.$ has zero twist).

$$
g_{\alpha \beta}^{\mathrm{sSCH}}=\hat{v}_{\alpha} \hat{v}_{\beta}-\hat{r}_{\alpha} \hat{r}_{\beta}-\hat{\vartheta}_{\alpha} \hat{\vartheta}_{\beta}-\hat{\varphi}_{\alpha} \hat{\varphi}_{\beta} .
$$

Here, $A^{2}=1-2 m_{0} / r$. The unit vectors are defined by

$$
\begin{array}{rlrl}
\hat{v}_{\alpha} \mathrm{d} x^{\alpha} & =A^{-1}\left(A^{2} \mathrm{~d} u+\mathrm{d} r\right), & & \hat{v}^{\alpha} \partial_{\alpha}=A^{-1}\left(\partial_{u}+\Omega \partial_{\varphi}\right), \\
\hat{r}_{\alpha} \mathrm{d} x^{\alpha}=A^{-1} \mathrm{~d} r, & & \hat{r}^{\alpha} \partial_{\alpha}=A^{-1}\left(\partial_{u}-A^{2} \partial_{r}+\Omega \partial_{\varphi}\right), \\
\hat{\vartheta}_{\alpha} \mathrm{d} x^{\alpha}=r \mathrm{~d} \vartheta, & & \hat{\vartheta}^{\alpha} \partial_{\alpha}=-r^{-1} \partial_{\vartheta}, \\
\hat{\varphi}_{\alpha} \mathrm{d} x^{\alpha}=r \sin \vartheta(\mathrm{d} \varphi-\Omega \mathrm{d} u), & & \hat{\varphi}^{\alpha} \partial_{\alpha}=-(r \sin \vartheta)^{-1} \partial_{\varphi} .
\end{array}
$$

The Einstein tensor is expanded as

$$
G_{\alpha \beta}^{\mathrm{sSCH}}=U^{2}\left(\hat{v}_{\alpha} \hat{v}_{\beta}-\hat{r}_{\alpha} \hat{r}_{\beta}+\hat{\vartheta}_{\alpha} \hat{\vartheta}_{\beta}+3 \hat{\varphi}_{\alpha} \hat{\varphi}_{\beta}\right)
$$

with $U^{2}=(9 / 4) \Omega^{2} \sin ^{2} \vartheta$. In the locally non-rotating frame, the energy-momentum tensor is

$$
T_{\alpha \beta}^{\mathrm{sSCH}}=\rho \hat{v}_{\alpha} \hat{v}_{\beta}+p_{r} \hat{r}_{\alpha} \hat{r}_{\beta}+p_{\vartheta} \hat{\vartheta}_{\alpha} \hat{\vartheta}_{\beta}+p_{\varphi} \hat{\varphi}_{\alpha} \hat{\varphi}_{\beta}
$$

It follows from $G_{\alpha \beta}=-8 \pi T_{\alpha \beta}$ that

$$
8 \pi \rho=-U^{2}, \quad 8 \pi p_{r}=U^{2}, \quad 8 \pi p_{\vartheta}=-U^{2}, \quad 8 \pi p_{\varphi}=-3 U^{2} .
$$

The Ricci tensor has the form

$$
R_{\alpha \beta}^{\mathrm{sSCH}}=2 U^{2}\left(\hat{v}_{\alpha} \hat{v}_{\beta}-\hat{r}_{\alpha} \hat{r}_{\beta}+\hat{\varphi}_{\alpha} \hat{\varphi}_{\beta}\right), \quad R_{\alpha \beta}^{\mathrm{sSCH}} l^{\beta}=2 U^{2} l_{\alpha},
$$

with $l^{\alpha} \partial_{\alpha}=\partial_{r}$ an eigenvector of the Ricci tensor.

The fluid velocity $\hat{v}^{\alpha}$ is expansion-free and twist-free, with shear scalar $\frac{1}{2} \sigma_{\alpha \beta} \sigma^{\alpha \beta}=U^{2}$ and acceleration $a^{\alpha}=\left(\frac{m_{0}}{A r^{2}}\right) \hat{r}^{\alpha}$. The content of spinning Schwarzschild can be interpreted as a rotating radial string fluid with $\rho+p_{r}=0$. 
The sSCH metric can be written as

$$
g_{\alpha \beta}^{\mathrm{sSCH}}=g_{\alpha \beta}^{\mathrm{SCH}}-2 \Omega l_{\alpha} S_{\beta},
$$

where $g_{\alpha \beta}^{\mathrm{SCH}}$ is the Schwarzschild metric and $S_{\beta}$ is a spacelike vector such that

$$
S_{\beta} \mathrm{d} x^{\beta}=(\Omega / 2) r^{2} \sin ^{2} \vartheta \mathrm{d} u-r^{2} \sin ^{2} \vartheta \mathrm{d} \varphi, \quad S_{\alpha} S^{\alpha}=-(r \sin \vartheta)^{2} .
$$

When $m_{0} \rightarrow 0$ in sSCH, we have spinning Minkowski (sM):

$$
g_{\alpha \beta}^{\mathrm{sM}}=\eta_{\alpha \beta}-2 \Omega l_{\alpha} S_{\beta} .
$$

This spacetime, as seen from null infinity, has angular momentum but no mass.

\subsection{Spinning Minkowski}

We set $m_{0}=0$ in $Z$ of equation (4) to obtain a metric with no global mass. The spinning Minkowski metric (sM) has one parameter, $r_{0}$, in function $\Omega(r)=r_{0}^{2} / r^{3}$.

$g_{\alpha \beta}^{\mathrm{sM}} \mathrm{d} x^{\alpha} \mathrm{d} x^{\beta}=\left(1-\Omega^{2} r^{2} \sin ^{2} \vartheta\right) \mathrm{d} u^{2}+2 \mathrm{~d} u \mathrm{~d} r+2 \Omega r^{2} \sin ^{2} \vartheta \mathrm{d} u \mathrm{~d} \varphi-r^{2}\left(\mathrm{~d} \vartheta^{2}+\sin ^{2} \vartheta \mathrm{d} \varphi^{2}\right)$.

The metric has two Killing vectors, stationary $k_{(\mathrm{u})}$ and axial $k_{(\varphi)}$. In terms of the Minkowski metric $\eta_{\alpha \beta}$, the sM metric can be written as

$$
g_{\alpha \beta}^{\mathrm{sM}} \mathrm{d} x^{\alpha} \mathrm{d} x^{\beta}=\eta_{\alpha \beta} \mathrm{d} x^{\alpha} \mathrm{d} x^{\beta}-\Omega^{2} r^{2} \sin ^{2} \vartheta \mathrm{d} u^{2}+2 \Omega r^{2} \sin ^{2} \vartheta \mathrm{d} u \mathrm{~d} \varphi .
$$

The rotation axis $[\vartheta: 0,2 \pi]$ is a regular line in the spacetime. As $r \rightarrow \infty, \Omega r^{2} \rightarrow 0$ and $g_{\alpha \beta}^{\mathrm{sM}} \rightarrow \eta_{\alpha \beta}$, hence the sM metric is asymptotically flat. In fact, one can remap the Minkowski metric

$$
\eta_{\alpha \beta} \mathrm{d} x^{\alpha} \mathrm{d} x^{\beta}=\mathrm{d} u^{2}+2 \mathrm{~d} u \mathrm{~d} r-r^{2}\left(\mathrm{~d} \vartheta^{2}+\sin ^{2} \vartheta \mathrm{d} \tilde{\varphi}^{2}\right)
$$

by shifting the $\tilde{\varphi}$ coordinate to $\tilde{\varphi}=\varphi-\Omega_{0} u$.

$\eta_{\alpha \beta} \mathrm{d} x^{\alpha} \mathrm{d} x^{\beta}=\left(1-\Omega_{0}^{2} r^{2} \sin ^{2} \vartheta\right) \mathrm{d} u^{2}+2 \mathrm{~d} u \mathrm{~d} r+2 \Omega_{0} r^{2} \sin ^{2} \vartheta \mathrm{d} u \mathrm{~d} \varphi-r^{2}\left(\mathrm{~d} \vartheta^{2}+\sin ^{2} \vartheta \mathrm{d} \varphi^{2}\right)$.

The Minkowski metric then coincides with the limit of the sM metric as $\Omega\left(\lim _{r \rightarrow \infty}\right) \rightarrow 0$, and $\Omega_{0} \rightarrow 0$.

The sM solution is written in the same locally non-rotating frame as $\mathrm{sSCH}$ above, but now the frame velocity $\hat{v}^{\alpha}$ is geodesic. It is also expansion-free and twist-free, with shear scalar $\frac{1}{2} \sigma_{\alpha \beta} \sigma^{\alpha \beta}=U^{2}=\left(3 r_{0}^{2} \sin \vartheta / 2 r^{3}\right)^{2}$.

$$
g_{\alpha \beta}^{\mathrm{sM}}=\hat{v}_{\alpha} \hat{v}_{\beta}-\hat{r}_{\alpha} \hat{r}_{\beta}-\hat{\vartheta}_{\alpha} \hat{\vartheta}_{\beta}-\hat{\varphi}_{\alpha} \hat{\varphi}_{\beta}
$$

The unit vectors are (this is the tetrad in equation (6) with $A=1$ )

$$
\begin{array}{llrl}
\hat{v}_{\alpha} \mathrm{d} x^{\alpha}=\mathrm{d} u+\mathrm{d} r, & \hat{v}^{\alpha} \partial_{\alpha}=\partial_{u}+\Omega \partial_{\varphi}, \\
\hat{r}_{\alpha} \mathrm{d} x^{\alpha}=\mathrm{d} r, & \hat{r}^{\alpha} \partial_{\alpha}=\partial_{u}-\partial_{r}+\Omega \partial_{\varphi}, \\
\hat{\vartheta}_{\alpha} \mathrm{d} x^{\alpha}=r \mathrm{~d} \vartheta, & \hat{\vartheta}^{\alpha} \partial_{\alpha}=-r^{-1} \partial_{\vartheta}, \\
\hat{\varphi}_{\alpha} \mathrm{d} x^{\alpha}=r \sin \vartheta(\mathrm{d} \varphi-\Omega \mathrm{d} u), & \hat{\varphi}^{\alpha} \partial_{\alpha}=-(r \sin \vartheta)^{-1} \partial_{\varphi} .
\end{array}
$$

The Einstein tensor and energy-momentum tensor here are the same as those of the sSCH solution above.

$G_{\alpha \beta}^{\mathrm{sM}}=U^{2}\left(\hat{v}_{\alpha} \hat{v}_{\beta}-\hat{r}_{\alpha} \hat{r}_{\beta}+\hat{\vartheta}_{\alpha} \hat{\vartheta}_{\beta}+3 \hat{\varphi}_{\alpha} \hat{\varphi}_{\beta}\right)$

$T_{\alpha \beta}^{\mathrm{sM}}=\rho \hat{v}_{\alpha} \hat{v}_{\beta}+p_{r} \hat{r}_{\alpha} \hat{r}_{\beta}+p_{\vartheta} \hat{\vartheta}_{\alpha} \hat{\vartheta}_{\beta}+p_{\varphi} \hat{\varphi}_{\alpha} \hat{\varphi}_{\beta}$,

$8 \pi \rho=-U^{2}, \quad 8 \pi p_{r}=U^{2}, \quad 8 \pi p_{\vartheta}=-U^{2}, \quad 8 \pi p_{\varphi}=-3 U^{2}$.

The sM solution contains a rotating radial string fluid with $\rho+p_{r}=0$. Since $U=$ $(3 / 2) r_{0}^{2} \sin \vartheta / r^{3}$, the entire matter content approaches vacuum as $r \rightarrow \infty$. 


\section{Mass}

The Bondi mass of $g^{\mathrm{sSCH}}$ is evaluated on a spherical cut of future null infinity $\mathcal{I}^{+}$. Equation (D.4) and the Weyl tensor component (equation (A.2)) yield

$$
M_{\mathrm{Bondi}}^{\mathrm{sSCH}}=-\frac{1}{8 \pi} \oint_{\partial \mathcal{N}}\left(\Psi_{2}^{0}+\bar{\Psi}_{2}^{0}\right) \sqrt{-g} \mathrm{~d} \vartheta \mathrm{d} \varphi=m_{0} .
$$

Since metric $g^{\mathrm{sM}}$ is the $m_{0} \rightarrow 0$ limit of $g^{\mathrm{sSCH}}$ it follows that

$$
M_{\text {Bondi }}^{\text {sM }}=0 \text {. }
$$

The Komar superpotential for Killing vector $k^{\beta}$ is

$$
U^{\alpha \beta}(k)=(-g)^{\frac{1}{2}}\left[\nabla^{\alpha} k^{\beta}-\nabla^{\beta} k^{\alpha}\right] .
$$

For timelike Killing vector $k_{(\mathrm{u})}^{\beta} \partial_{\beta}=\partial_{u}$ one writes $U^{\alpha \beta}\left(\partial_{u}\right)$, and for axial Killing vector $k_{(\varphi)}^{\beta} \partial_{\beta}=\partial_{\varphi}$ one writes $U^{\alpha \beta}\left(\partial_{\varphi}\right)$. Metrics $g^{\mathrm{sSCH}}$ and $g^{\mathrm{sM}}$ both have $(-g)^{\frac{1}{2}}=r^{2} \sin \vartheta$. For $u=$ const 3 -surface $\mathcal{N}$, the mass within a $u=$ const, $r=$ const 2 -surface $\partial \mathcal{N}$ is

$$
M_{\mathrm{Komar}}=-\frac{1}{8 \pi} \oint_{\partial \mathcal{N}} U^{\alpha \beta}\left(\partial_{u}\right) \mathrm{d} S_{\alpha \beta} .
$$

Metric $g_{\alpha \beta}^{\mathrm{sSCH}}$ and 2-surface $\mathrm{d} S_{\alpha \beta}=u_{,[\alpha} r_{, \beta]} \mathrm{d} \vartheta \mathrm{d} \varphi$ contains global mass $m_{0}$ and quasilocal mass at all $r$ beyond the ergosphere.

$$
\begin{aligned}
& M_{\mathrm{Komar}}^{\mathrm{sSCH}}=-\frac{1}{8 \pi} \int_{0}^{2 \pi} \int_{0}^{\pi}\left(-2 m_{0} \sin \vartheta-3 \frac{r_{0}^{4}}{r^{3}} \sin ^{3} \vartheta\right) \mathrm{d} \vartheta \mathrm{d} \varphi=m_{0}+\frac{r_{0}^{4}}{r^{3}}, \\
& M_{\mathrm{Komar}}^{\mathrm{sM}}=\frac{r_{0}^{4}}{r^{3}} .
\end{aligned}
$$

There is no global mass for $g^{\mathrm{sM}}$ since $m_{0}=0$ and $M_{\mathrm{Komar}}^{\mathrm{sM}} \rightarrow 0$ as $r \rightarrow \infty$.

Although the sM solution is not spherically symmetric, we can compute a sectional curvature mass since tetrad vectors $\hat{\vartheta}^{\alpha}$ and $\hat{\varphi}^{\alpha}$ of equations $(17 c)$ and $(17 d)$ form a bivector which satisfies the Frobenius surface-forming condition. They span a family of closed 2-surfaces. The sectional curvature (Gaussian curvature) mass is

$$
-\frac{2 M_{\mathrm{curv}}^{\mathrm{sSCH}}}{r^{3}}=R_{\alpha \beta \mu \nu} \hat{\vartheta}^{\alpha} \hat{\varphi}^{\beta} \hat{\vartheta}^{\mu} \hat{\varphi}^{\nu}=m_{0}
$$

The sectional curvature mass for $g^{\mathrm{sM}}$ vanishes.

The explanation of why $M^{\mathrm{sSCH}}$ and $M^{\mathrm{sM}}$ are positive when the density $\rho=-U^{2} / 8 \pi$ is negative lies with the relativistic contribution of pressure to mass. If one traces the Komar integral back it then becomes clear.

$$
\begin{aligned}
M_{\text {Komar }} & =-\frac{1}{8 \pi} \oint_{\partial \mathcal{N}} U^{\alpha \beta}\left(\partial_{u}\right) \mathrm{d} S_{\alpha \beta} \\
& =-\frac{1}{8 \pi} \oint_{\partial \mathcal{N}} \sqrt{-g} k_{(\mathrm{u})}^{[\alpha ; \beta]} \mathrm{d} S_{\alpha \beta} \\
& =-\frac{1}{8 \pi} \int_{\mathcal{N}} \sqrt{-g} k_{(\mathrm{u})}^{\alpha} R_{\alpha}{ }^{\beta} \mathrm{d} S_{\beta}
\end{aligned}
$$

for $\mathrm{d} S_{\beta}=u_{, \beta} \mathrm{d}^{3} x$. The 3-volume integration is over any $u=$ const null surface from $r$ (beyond the ergosurface) to $\infty$. The Ricci tensor provides

$$
\begin{aligned}
M_{\text {Komar }} & =-\frac{1}{8 \pi} \int_{\mathcal{N}} \sqrt{-g}\left(-2 \rho-p_{r}+p_{\varphi}\right) \mathrm{d}^{3} x \\
& =\frac{1}{4 \pi} \int_{\mathcal{N}} \sqrt{-g} U^{2} \mathrm{~d} r \mathrm{~d} \vartheta \mathrm{d} \varphi .
\end{aligned}
$$

For $g^{\mathrm{sSCH}}$ the volume integral does not measure the $m_{0}$ contribution. 


\section{Angular momentum}

For $u=$ const 3 -surface $\mathcal{N}$, the angular momentum within a $u=$ const, $r=$ const 2 -surface $\partial \mathcal{N}$ is

$$
J=-\frac{1}{16 \pi} \oint_{\partial \mathcal{N}} U^{\alpha \beta}\left(\partial_{\varphi}\right) \mathrm{d} S_{\alpha \beta}
$$

Metric $g^{\mathrm{sSCH}}$ and metric $g^{\mathrm{sM}}$ admit constant $J$ at all $r$ beyond the ergosphere. For 2-surface element $\mathrm{d} S_{\alpha \beta}=u_{,[\alpha} r_{, \beta]} \mathrm{d} \vartheta \mathrm{d} \varphi$

$$
J^{\mathrm{sSCH}}=J^{\mathrm{sM}}=-\frac{1}{16 \pi} \int_{0}^{2 \pi} \int_{0}^{\pi}\left(3 r_{0}^{2} \sin ^{3} \vartheta\right) \mathrm{d} \vartheta \mathrm{d} \varphi=-\frac{1}{2} r_{0}^{2} .
$$

This is a global value.

The normalization of the Komar integrals is chosen [9] so that

$$
J^{\operatorname{Kerr}}\left(\partial_{\varphi}\right)=-m_{0} a \quad \text { and } \quad M^{\operatorname{Kerr}}\left(\partial_{u}\right)=m_{0} .
$$

The sign difference occurs because our -2 signature gives timelike vectors positive norms and spacelike vectors negative norms.

\section{Surfaces of sM}

\subsection{Horizon}

Spacelike closed 2-surfaces are trapped if their null generators (incoming and outgoing null geodesics) converge towards the future. The time evolution of the outermost trapped surface is a null surface called the 'apparent horizon'. The spherical 2-surfaces of metric $g^{\mathrm{sM}}$ have area $4 \pi r^{2}$, with null geodesic generators $l^{\alpha}$ and $n^{\alpha}$ (see equation (A.4)). Their expansions (convergences) are given, respectively, by

$$
\rho=-l^{\alpha} \partial_{\alpha}(\ln r)=-1 / r, \quad \mu=n^{\alpha} \partial_{\alpha}(\ln r)=-1 /(2 r) .
$$

(here $\rho$ is a spin coefficient, not mass density). There can be no horizon since neither $\rho$ nor $\mu$ change sign over the interval $0<r<\infty$.

\subsection{Ergosurface}

The norm of the timelike Killing vector is, with $\Omega=r_{0}^{2} / r^{3}$,

$$
\partial_{u}^{\mathrm{sM}} \cdot \partial_{u}^{\mathrm{sM}}=1-\Omega^{2} r^{2} \sin ^{2} \vartheta
$$

placing the ergosphere at $r^{2}= \pm r_{0}^{2} \sin \vartheta=\left|r_{0}^{2} \sin \vartheta\right|$.

The shape of the ergosurface (outer boundary of the ergosphere) is determined by the locus of points where the norm of $\partial_{u}^{\mathrm{sM}}$ is zero, $r^{2}=\left|r_{0}^{2} \sin \vartheta\right|$. The curve ranges from $r=0$ to $r=r_{0}$, and is called the 'lemniscate of Bernoulli' [10]. In $(x, y, z)$ coordinates, with rotation about the $z$-axis, the ergosurface is shaped like a deformed torus without a centre hole, since the surface boundary curve goes smoothly to 0 at $x=y=z=0$. Inside the ergosurface the spacelike 2-surfaces are 2-spheres.

With $\partial_{u}^{\mathrm{sM}} \cdot \partial_{u}^{\mathrm{sM}}<0$, the Gaussian curvature of $\vartheta, \varphi$ 2-surfaces inside the ergosphere is

$$
\mathcal{K}_{\text {ergo }}=1 / r^{2} \text {. }
$$

We compute the Euler-Poincaré characteristic $\chi$ of the ergosurface. The 2-surface metric is

$$
\mathrm{d} s_{2-\text { surf }}^{2}=r_{0}^{2} \sin \vartheta\left(\mathrm{d} \vartheta^{2}+\sin ^{2} \vartheta \mathrm{d} \varphi^{2}\right)
$$


and

$$
\mathcal{K}_{2 \text {-ergo }}=\frac{1}{r_{0}^{2} \sin \vartheta}
$$

undefined at the poles $\vartheta=0, \pi$. The Euler-Poincaré characteristic [11] is then

$$
2 \pi \chi=\int_{0}^{2 \pi} \int_{0}^{\pi} \mathcal{K}_{2 \text {-ergo }}\left(r_{0}^{2} \sin ^{2} \vartheta\right) \mathrm{d} \vartheta \mathrm{d} \varphi=4 \pi .
$$

Thus $\chi=2$, with associated genus $g=0$ which characterizes the topology of a standard 2-sphere.

The Kerr ergosphere is quite different. The norm of the Kerr timelike Killing vector (in Boyer-Lindquist coordinates) is

$$
\partial_{t}^{\text {Kerr }} \cdot \partial_{t}^{\text {Kerr }}=1-\frac{2 m_{0} r}{r^{2}+a^{2} \cos ^{2} \vartheta} .
$$

The Killing vector is spacelike in the region $2 m_{0} \leqslant r<m_{0}+\left(m_{0}^{2}-a^{2} \cos ^{2} \vartheta\right)^{1 / 2}$. The Kerr ergosurface is tangent to the rotating trapped surface at the poles.

\subsection{Geodesic deviation of $s M$}

Test particles move along a congruence of timelike geodesics $v^{\alpha}=\mathrm{d} x^{\alpha}(\tau) / \mathrm{d} \tau$. Vector $v^{\alpha}$ is twist-free and acceleration-free with respect to metric $g^{\mathrm{sM}}$ and is given by $v^{\alpha} \partial_{\alpha}=\partial_{u}+\Omega \partial_{\varphi}$. Covariant differentiation along the geodesics is defined by

$$
\mathrm{D} / \mathrm{d} \tau:=v^{\alpha} \nabla_{\alpha} .
$$

Deviation vector $\eta^{\alpha}$ is tangent to the line connecting pairs of neighbouring geodesics in the congruence. It is Lie transported along the congruence and satisfies

$$
\mathcal{L}_{v} \eta^{\alpha}=0 .
$$

$\frac{\mathrm{D}^{2}}{\mathrm{~d} \tau^{2}} \eta^{\alpha}$ gives the relative acceleration between neighbouring test particles. The geodesic deviation equation is $[12,13]$

$$
\frac{\mathrm{D}^{2}}{\mathrm{~d} \tau^{2}} \eta^{\alpha}=\left[R_{\beta \mu \nu}^{\alpha} v^{\beta} v^{\mu}\right] \eta^{\nu} .
$$

If one considers any $\varphi=$ const slice of a family of $\{\vartheta, \varphi\} 2$-spheres then $\eta=(1 / \sin \vartheta) \partial_{\varphi}$ is a deviation vector linking timelike geodesics orthogonal to the 2 -spheres. We find

$$
\frac{\mathrm{D}^{2}}{\mathrm{~d} \tau^{2}} \eta^{\alpha}=\left(\frac{9}{4} \Omega^{2} r\right) \hat{\varphi}^{\alpha}
$$

for $\Omega=r_{0}^{2} / r^{3}$ and tetrad vector (17d). A view of the geodesic congruence shows the tip of the connecting vector $\eta$ spiralling up the congruence with acceleration $\Omega^{2} r$.

\section{Discussion}

A method has been presented for adding angular momentum to static spacetimes. Metric $g^{\mathrm{sSCH}}$ is a spinning generalization of the vacuum Schwarzschild metric. The spin-up method creates a spinning object with an atmosphere in an asymptotically flat spacetime. There are many isolated astrophysical systems in our observable universe that spin. We have recently seen that the unexpected behaviour of the angular velocity of globular clusters was a strong clue to the existence of dark matter and dark energy. Analytic metrics, with a family of possible angular 
velocities, can be a useful tool for studying and classifying rotating systems. They can be used to predict and analyse observable data such as lensing and to check numerical simulations.

Here we started with the Schwarzschild metric and added a rotation term, maintaining Bondi-Sachs metric form and using the rotation term from the linearized Kerr metric (transformed to a Bondi-Sachs frame) as a guide. The resulting metric, $g^{\mathrm{sSCH}}$, has global mass and angular momentum. The global mass is $M_{\mathrm{Bondi}}^{\mathrm{sSCH}}=m_{0}$, the Bondi mass. The Komar mass of spinning Schwarzschild $M_{\mathrm{Komar}}^{\mathrm{sSCH}}=m_{0}+r_{0}^{4} / r^{3}$ has both global mass and a quasilocal term which falls off asymptotically. When $m_{0}$ is set to zero, then $g^{\mathrm{sSCH}} \rightarrow g^{\mathrm{sM}}$ the spinning Minkowski metric.

The $g^{\mathrm{sM}}$ metric has unusual properties. Unusual because there is quasilocal mass but no global mass, and there is an ergosphere but no event horizon. Values from the Komar superpotential provide global angular momentum, $J^{\mathrm{sM}}=-\frac{1}{2} r_{0}^{2}$. The quasilocal mass does fall off asymptotically. It arises from the kinetic energy of the rotating string fluid. As seen from conformal infinity, $g^{\mathrm{sM}}$ is a large spinning object with no total weight.

In this first work we focused on asymptotically flat seed metrics, and spun them up in a prescribed manner. Future work will allow other configurations, such as cylindrical symmetry, or extended matter, and the Bondi-Sachs metric form can be relaxed.

\section{Appendix A. Null tetrad}

\section{A.1. Spinning Schwarzschild}

The null tetrad which spans metric $g_{\alpha \beta}^{\mathrm{sSCH}}$ of equation (4) is, with $\Omega=r_{0}^{2} / r^{3}$ and $A^{2}=1-2 m_{0} / r$

$$
\begin{array}{ll}
l_{\mu} \mathrm{d} x^{\mu}=\mathrm{d} u, & l^{\mu} \partial_{\mu}=\partial_{r}, \\
n_{\mu} \mathrm{d} x^{\mu}=\left(A^{2} / 2\right) \mathrm{d} u+\mathrm{d} r, & n^{\mu} \partial_{\mu}=\partial_{u}-\left(A^{2} / 2\right) \partial_{r}+\Omega \partial_{\varphi}, \\
m_{\mu} \mathrm{d} x^{\mu}=\left(\frac{\mathrm{i} \Omega r \sin \vartheta}{\sqrt{2}}\right) \mathrm{d} u-\left(\frac{r}{\sqrt{2}}\right)(\mathrm{d} \vartheta+\mathrm{i} \sin \vartheta \mathrm{d} \varphi), & m^{\mu} \partial_{\mu}=\frac{1}{\sqrt{2} r}\left(\partial_{\vartheta}+\frac{\mathrm{i}}{\sin \vartheta} \partial_{\varphi}\right) .
\end{array}
$$

The Weyl tensor components are

$$
\begin{array}{ll}
\Psi_{0}=0, & \Psi_{1}=\left(\frac{3 i}{2 \sqrt{2}}\right) \frac{\Omega \sin \vartheta}{r}, \\
\Psi_{2}=-\frac{m_{0}}{r^{3}}-\frac{3}{2}\left(\Omega^{2} \sin ^{2} \vartheta+\mathrm{i} \frac{\Omega}{r} \cos \vartheta\right), & \Psi_{3}=-\frac{\Psi_{1}}{2}\left(1-\frac{2 m_{0}}{r}\right), \\
\Psi_{4}=0 . &
\end{array}
$$

The Ricci components are

$$
\begin{aligned}
& \Phi_{00}=\Phi_{01}=\Phi_{12}=\Phi_{22}=0, \quad \Phi_{02}=\frac{9}{8} \Omega^{2} \sin ^{2} \vartheta, \\
& \Phi_{11}=-\frac{3}{2} \Phi_{02}, \quad 6 \Lambda=\mathcal{R} / 4=\Phi_{02} .
\end{aligned}
$$

\section{A.2. Spinning Minkowksi}

The massless spinning metric, with $\Omega=r_{0}^{2} / r^{3}$

$g_{\alpha \beta}^{\mathrm{sM}} \mathrm{d} x^{\alpha} \mathrm{d} x^{\beta}=\left(1-\Omega^{2} r^{2} \sin ^{2} \vartheta\right) \mathrm{d} u^{2}+2 \mathrm{~d} u \mathrm{~d} r+2 \Omega r^{2} \sin ^{2} \vartheta \mathrm{d} u \mathrm{~d} \varphi-r^{2}\left(\mathrm{~d} \vartheta^{2}+\sin ^{2} \vartheta \mathrm{d} \varphi^{2}\right)$ 
is expanded in a null tetrad as

$$
g_{\alpha \beta}^{\mathrm{sM}}=l_{\alpha} n_{\beta}+n_{\alpha} l_{\beta}-m_{\alpha} \bar{m}_{\beta}-\bar{m}_{\alpha} m_{\beta}
$$

where

$l_{\alpha} \mathrm{d} x^{\alpha}=\mathrm{d} u$

$$
\begin{array}{ll}
l_{\alpha} \mathrm{d} x^{\alpha}=\mathrm{d} u, & l^{\alpha} \partial_{\alpha}=\partial_{r}, \\
n_{\alpha} \mathrm{d} x^{\alpha}=\mathrm{d} u / 2+\mathrm{d} r, & n^{\alpha} \partial_{\alpha}=\partial_{u}-(1 / 2) \partial_{r}+\Omega \partial_{\varphi}, \\
m_{\alpha} \mathrm{d} x^{\alpha}=\frac{\mathrm{i}}{\sqrt{2}}(\Omega r \sin \vartheta) \mathrm{d} u-\frac{r}{\sqrt{2}}(\mathrm{~d} \vartheta+\mathrm{i} \sin \vartheta \mathrm{d} \varphi), & m^{\alpha} \partial_{\alpha}=\frac{1}{\sqrt{2} r}\left(\partial_{\vartheta}+\frac{\mathrm{i}}{\sin \vartheta} \partial_{\varphi}\right) .
\end{array}
$$

$n_{\alpha} \mathrm{d} x^{\alpha}=\mathrm{d} u / 2+\mathrm{d} r$

This tetrad has six zero-valued spin coefficients: $\kappa=\epsilon=\sigma=\lambda=\nu=\gamma=0$. The others are

$$
\begin{array}{ll}
\rho=-\frac{1}{r}, & \mu=-\frac{1}{2 r}, \\
\pi=\left(\frac{3 \mathrm{i}}{2 \sqrt{2}}\right) \Omega \sin \vartheta, & \tau=-\pi, \\
\beta=\frac{1}{2 \sqrt{2}}\left(\frac{\cot \vartheta}{r}-\frac{3 \mathrm{i}}{2} \Omega \sin \vartheta\right), & \alpha=-\beta .
\end{array}
$$

$l^{\alpha}$ is geodesic since $\kappa=0$, and $n^{\alpha}$ is geodesic since $v=0$.

The Weyl tensor components are

$$
\begin{array}{rlrl}
\Psi_{0} & =0, & \Psi_{1} & =\left(\frac{3 i}{2 \sqrt{2}}\right) \frac{\Omega \sin \vartheta}{r}, \\
\Psi_{2} & =-\frac{3}{2}\left(\Omega^{2} \sin ^{2} \vartheta+i \frac{\Omega}{r} \cos \vartheta\right), & \Psi_{3} & =-\Psi_{1} / 2, \\
\Psi_{4} & =0 .
\end{array}
$$

If there was a Bondi mass, it would appear in $\Psi_{2}$ at $O\left(1 / r^{3}\right)$ since $r$ is a valid luminosity distance. The Einstein tensor null tetrad components are

$G_{\alpha \beta}^{\mathrm{sM}}=-4 \Phi_{11}\left(l_{\alpha} n_{\beta}+n_{\alpha} l_{\beta}\right)-2 \Phi_{02}\left(m_{\alpha} m_{\beta}+\bar{m}_{\alpha} \bar{m}_{\beta}\right)+\left(2 \Phi_{11}-\mathcal{R} / 4\right) g_{\alpha \beta}^{\mathrm{sM}}$

where

$$
\begin{array}{ll}
\Phi_{00}=\Phi_{01}=\Phi_{12}=\Phi_{22}=0, & \Phi_{02}=\frac{9}{8} \Omega^{2} \sin ^{2} \vartheta=\frac{U^{2}}{2}, \\
\Phi_{11}=-\frac{3}{2} \Phi_{02}, & 6 \Lambda=\mathcal{R} / 4=\Phi_{02} .
\end{array}
$$

One can transform from the null tetrad to the locally non-rotating tetrad in equation (17):

$\hat{v}=l / 2+n, \quad \hat{r}=-l / 2+n, \quad \hat{\vartheta}=-(m+\bar{m}) / \sqrt{2}, \quad \hat{\varphi}=\mathrm{i}(m-\bar{m}) / \sqrt{2}$.

\section{Appendix B. Invariants}

In general, the quadratic Riemann invariant (Kretschmann scalar) is related to the Weyl and Ricci invariants by

$$
R_{\alpha \beta \mu \nu} R^{\alpha \beta \mu \nu}=C_{\alpha \beta \mu \nu} C^{\alpha \beta \mu \nu}+2 R_{\alpha \beta} R^{\alpha \beta}-\mathcal{R}^{2} / 3
$$


The quadratic invariants for $g^{\mathrm{sSCH}}$ are, with $\Omega=r_{0}^{2} / r^{3}$,

$$
\begin{aligned}
& R_{\alpha \beta \mu \nu}^{\mathrm{sSCH}} R_{\mathrm{sSCH}}^{\alpha \beta \mu \nu}=48 \frac{m_{0}^{2}}{r^{6}}+24 \frac{m_{0}}{r^{3}}(3 \Omega \sin \vartheta)^{2}+R_{\alpha \beta \mu \nu}^{\mathrm{sM}} R_{\mathrm{sM}}^{\alpha \beta \mu \nu}, \\
& C_{\alpha \beta \mu \nu}^{\mathrm{sSCH}} C_{\mathrm{sSCH}}^{\alpha \beta \mu \nu}=48 \frac{m_{0}^{2}}{r^{6}}+24 \frac{m_{0}}{r^{3}}(3 \Omega \sin \vartheta)^{2}+C_{\alpha \beta \mu \nu}^{\mathrm{sM}} C_{\mathrm{sM}}^{\alpha \beta \mu \nu}, \\
& R_{\alpha \beta}^{\mathrm{sSCH}} R_{\mathrm{sSCH}}^{\alpha \beta}=\frac{243}{4} \Omega^{4} \sin ^{4} \vartheta, \quad \mathcal{R}_{\mathrm{sSCH}}^{2}=\frac{81}{4} \Omega^{4} \sin ^{4} \vartheta,
\end{aligned}
$$

and the quadratic invariants for $g^{\mathrm{sM}}$ are

$$
\begin{aligned}
& R_{\alpha \beta \mu \nu}^{\mathrm{sM}} R_{\mathrm{sM}}^{\alpha \beta \mu \nu}=-3\left(\frac{6 \Omega}{r}\right)^{2}+2\left(\frac{6 \Omega \sin \vartheta}{r}\right)^{2}+\frac{9 \times 99}{4} \Omega^{4} \sin ^{4} \vartheta, \\
& C_{\alpha \beta \mu \nu}^{\mathrm{sM}} C_{\mathrm{sM}}^{\alpha \beta \mu \nu}=-3\left(\frac{6 \Omega}{r}\right)^{2}+2\left(\frac{6 \Omega \sin \vartheta}{r}\right)^{2}+108 \Omega^{4} \sin ^{4} \vartheta, \\
& R_{\alpha \beta}^{\mathrm{sM}} R_{\mathrm{sM}}^{\alpha \beta}=\frac{243}{4} \Omega^{4} \sin ^{4} \vartheta, \quad \mathcal{R}_{\mathrm{sM}}^{2}=\frac{81}{4} \Omega^{4} \sin ^{4} \vartheta .
\end{aligned}
$$

For comparison, the Kerr invariant is

$$
\begin{aligned}
R_{\alpha \beta \mu \nu}^{\mathrm{Kerr}} R_{\mathrm{Kerr}}^{\alpha \beta \mu \nu} & =C_{\alpha \beta \mu \nu}^{\mathrm{Kerr}} C_{\text {Kerr }}^{\alpha \beta \mu \nu} \\
& =48 \frac{m_{0}^{2}}{r^{6}} \frac{1-\alpha^{2} \cos ^{2} \vartheta}{\left(1+\alpha^{2} \cos ^{2} \vartheta\right)^{6}}\left(1-14 \alpha^{2} \cos ^{2} \vartheta+\alpha^{4} \cos ^{4} \vartheta\right)
\end{aligned}
$$

where $\alpha=a / r$.

\section{Appendix C. Poincaré inequality}

An inequality due to Poincaré [14] shows that $\Omega$ is reasonable. Consider the equilibrium of a rotating, self-gravitating fluid obeying Newtonian gravity.

$$
\nabla^{2} \phi=4 \pi G \rho
$$

for uniform density $\rho$. The fluid is spinning with constant $\Omega$ about the $\hat{z}$-axis. The force on the liquid is

$$
\vec{F}=-\nabla\left[\phi-\left(\Omega^{2} / 2\right)\left(x^{2}+y^{2}\right)\right]
$$

For equilibrium, the total force on the boundary of fluid volume $V$ with outward normal $\hat{n}$ must be

$$
\begin{aligned}
\oint \vec{F} \cdot \hat{n} \mathrm{~d}^{2} x \leqslant 0 & \Rightarrow-\oint \nabla\left[\phi-\left(\Omega^{2} / 2\right)\left(x^{2}+y^{2}\right)\right] \cdot \hat{n} \mathrm{~d}^{2} x \leqslant 0 \\
& \Rightarrow-\int \nabla^{2}\left[\phi-\left(\Omega^{2} / 2\right)\left(x^{2}+y^{2}\right)\right] \mathrm{d}^{3} x \leqslant 0 \Rightarrow \int\left(4 \pi G \rho-2 \Omega^{2}\right) \mathrm{d}^{3} x \geqslant 0 .
\end{aligned}
$$

The mass, volume and average density are

$$
M=\int \rho \mathrm{d}^{3} x, \quad V=\int \mathrm{d}^{3} x, \quad \bar{\rho}=M / V
$$

and so

$$
\Omega^{2} \leqslant 2 \pi G \bar{\rho} .
$$

It is simple to verify that $\Omega(r)=r_{0}^{2} / r^{3}$ satisfies Poincaré's inequality for $r>r_{0}$ and quasilocal mass $M=r_{0}^{4} / r^{3}$ in equation (25). The average density is $\bar{\rho}=M /\left(4 \pi r^{3} / 3\right)=3 r_{0}^{4} / 4 \pi$ and $\Omega^{2}=r_{0}^{4} / r^{6}$. 


\section{Appendix D. Bondi-Sachs metric}

Asymptotically flat systems are described by the Bondi-Sachs metric

$g_{\mu \nu}^{\mathrm{BS}} \mathrm{d} x^{\mu} \mathrm{d} x^{\nu}=\frac{V \mathrm{e}^{2 b}}{r} \mathrm{~d} u^{2}+2 \mathrm{e}^{2 b} \mathrm{~d} u \mathrm{~d} r-r^{2} H_{A B}\left(\mathrm{~d} x^{A}-U^{A} \mathrm{~d} u\right)\left(\mathrm{d} x^{B}-U^{B} \mathrm{~d} u\right)$,

where outgoing null hypersurfaces are labelled by $u$ and coordinates are $\left(x^{0}=u, x^{1}=r, x^{2}=\right.$ $\left.\vartheta, x^{3}=\varphi\right)$. The Bondi-Sachs metric [15] extends Bondi's original metric [16] to include $\varphi$ dependence and has six independent functions $\left\{V, b, U^{\vartheta}, U^{\varphi}, y, q\right\}$ of $(u, r, \vartheta, \varphi)$. The rays of each $u=$ const null surface are null geodesics $x^{\alpha}(r)$ with tangent $\mathrm{d} x^{\alpha} / \mathrm{d} r$ where $x^{1}=r$ is a luminosity parameter. Coordinates $(\vartheta, \varphi)$ are constant along each ray. The luminosity parameter is defined by $r^{4} \sin ^{2} \vartheta=\operatorname{det}\left(g_{A B}\right)=\operatorname{det}\left(r^{2} H_{A B}\right)$ where

$$
H_{A B}=\left[\begin{array}{cc}
\mathrm{e}^{2 y} \cosh (2 q) & \sinh (2 q) \sin \vartheta \\
\sinh (2 q) \sin \vartheta & \mathrm{e}^{-2 y} \cosh (2 q) \sin ^{2} \vartheta
\end{array}\right] .
$$

The boundary conditions on the metric functions in the limit of future null infinity $\mathcal{I}^{+}$are

$$
r U^{A} \rightarrow 0, \quad b \rightarrow 0, \quad y \rightarrow 0, \quad q \rightarrow 0, \quad V / r \rightarrow 1 .
$$

Notation was chosen to avoid confusion between Sachs metric functions and Newman-Penrose spin coefficients:

$$
2 y=\gamma+\delta \quad \text { (Sachs), } \quad 2 q=\gamma-\delta \quad \text { (Sachs), } \quad b=\beta \quad \text { (Sachs). }
$$

The $u=$ const hypersurfaces have null geodesic tangent $l^{\alpha} \partial_{\alpha}$ which is also hypersurface orthogonal as $l_{\alpha} \mathrm{d} x^{\alpha}=\mathrm{d} u$. The twist of $l^{\alpha}$ is zero and its expansion and shear are given by

$$
\rho=-\mathrm{e}^{-2 b} / r, \quad \sigma=-\mathrm{e}^{-2 b}\left[\left(\partial_{r} y\right) \cosh (2 q)+\mathrm{i}\left(\partial_{r} q\right)\right]
$$

which follows from $r$ as a luminosity parameter, and where the phase of $\sigma$ is determined by the choice of tetrad orientation. The Bondi mass aspect $M(u, \vartheta, \varphi)$ is

$$
-2 M=\Psi_{2}^{0}+\bar{\Psi}_{2}^{0}+\partial_{u}\left(\sigma^{0} \bar{\sigma}^{0}\right),
$$

where the zero superscript indicates the leading coefficient in a $1 / r$ expansion. The Bondi mass is the 2-surface integral of the mass aspect over a topological 2-sphere at $\mathcal{I}^{+}$

$$
M_{\text {Bondi }}=-\frac{1}{8 \pi} \oint_{S^{2}}\left[\Psi_{2}^{0}+\bar{\Psi}_{2}^{0}+\partial_{u}\left(\sigma^{0} \bar{\sigma}^{0}\right)\right] \sqrt{-g} \mathrm{~d} \vartheta \mathrm{d} \varphi
$$

\section{References}

[1] Newman E T and Janis A I 1965 J. Math. Phys. 6915

[2] Newman E T, Couch T E, Chinnapared K, Exton A, Prakash A and Torrence R 1965 J. Math. Phys. 6918

[3] Newman E T 1973 J. Math. Phys. 14774

[4] Drake S P and Turalla R 1997 Class. Quantum Grav. 141883

[5] Ehlers J 1962 Les Théories Relativistes de la Gravitation ed A Lichnerowicz and M A Tonnelat (Coll. Int. CNRS No 91) (Paris: CNRS)

[6] Geroch R 1971 J. Math. Phys. 12918

[7] Geroch R 1972 J. Math. Phys. 13394

[8] Bicak J and Pravdova A 1998 J. Math. Phys. 346011

[9] Glass E N and Naber M G 1994 J. Math. Phys. 354178

[10] Lawrence J D 1999 A Catalog of Special Plane Curves (New York: Dover)

[11] do Carmo M P 1976 Differential Geometry of Curves and Surfaces (NJ: Prentice-Hall)

[12] Synge J L 1964 Relativity: The General Theory (Amsterdam: North-Holland)

[13] Szekeres P 1965 J. Math. Phys. 61387

[14] Poincaré H 1885 Acta Math. 7259

[15] Sachs R K 1962 Proc. R. Soc. A 270103

[16] Bondi H, van der Burg M and Metzner A 1962 Proc. R. Soc. A 26921 